\title{
Periventricular leucomalacia and intraventricular haemorrhage in the preterm neonate
}

\author{
J Q TROUNCE, N RUTTER, AND M I LEVENE \\ Department of Child Health, Leicester University Medical School, and Department of Neonatal Medicine and \\ Surgery, City Hospital, Nottingham
}

SUMmaRY Two hundred very low birthweight infants were prospectively scanned to ascertain the incidence of periventricular leucomalacia (PVL) and haemorrhage. Before collection of data, clear definitions of ultrasound abnormalities believed to represent PVL and intraventricular haemorrhage were described. These referred to small and moderate intraventricular haemorrhage, parenchymal haemorrhage, and PVL, including prolonged flare (echoes in the periventricular region lasting for two weeks or more and not becoming cystic). Sixty nine infants (34\%) had no abnormality on ultrasound scans. Intraventricular haemorrhage occurred in 107 babies ( 37 grade I and 62 grade II), and only eight infants were thought to have true parenchymal haemorrhage. Ultrasound appearances of PVL were seen in 27 infants, 19 of whom developed cysts and eight died in the precystic stage. Prolonged flare occurred in another 25 babies. Unilateral parenchymal haemorrhage occurred in four infants who subsequently developed cystic PVL in the contralateral hemisphere. Twenty one infants developed ventricular dilatation, 12 of whom had associated parenchymal-lesions. Haemorrhage, PVL, and flare occurred commonly in infants of 30 weeks' gestation and below and became markedly less common in more mature infants. We believe prolonged flare represents a form of PVL, and in this study a total of 52 $(26 \%)$ infants had an ultrasound appearance of periventricular leucomalacia, an incidence considerably higher than previously reported.

Haemorrhage in and around the lateral ventricles is a common lesion of the preterm brain, with an incidence variously reported between $32 \%^{1}$ and $90 \%{ }^{2}$ Periventricular leucomalacia (PVL) has also been recognised as affecting the infant's brain, but its incidence has been largely derived from postmortem studies and may not reflect its incidence in surviving infants. High resolution real time ultrasound enables a diagnosis of PVL to be made in the living infant, ${ }^{3-11}$ but there have been only three studies reporting its overall incidence, ${ }^{45} 11$ which ranges between $7 \cdot 5 \%$ and $17 \cdot 8 \%$ of low birthweight populations. Preliminary data suggest that PVL is associated with a poor prognosis ${ }^{6} 7^{12-14}$ and for this reason deserves more careful study. No agreed definitions exist for the ultrasound appearances of PVL, and as a preliminary to our study we carefully described the appearances that we believed represented ischaemic brain lesions. We then prospectively examined 200 very low birthweight infants to define the incidence of this condition, assess patho- logical correlation with scan findings, and look at risk factors for development of PVL and its effect on neurodevelopmental outcome. This paper compares the incidence of PVL with intraventricular haemorrhage in a group of infants weighing $1500 \mathrm{~g}$ or less at birth.

\section{Patients and methods}

Over a 16 month period (1 January 1984-30 April 1985 ) all neonates of birth weight $\leqslant 1500 \mathrm{~g}$ who were admitted to two neonatal intensive care units (Leicester Royal Infirmary and Nottingham City Hospital) were scanned at least twice each week for the first month and then once each week until discharge. All babies with an abnormality on the predischarge scan were recalled for at least one further scan. In both centres an ATL Mark III real time sector scanner fitted with a $3.5,5$, and $7.5 \mathrm{MHz}$ multifrequency scanhead was used, and all scans were performed by the same observer (JQT). A 
permanent record of all scans was kept on either videotape or $x$ ray film, and abnormalities were reviewed by another of the authors (MIL).

Definition of scan abnormalities. At the start of the study we clearly defined the ultrasound abnormalities we had consistently recognised over the previous three years. These definitions were adhered to throughout the study and final diagnosis was made at discharge or death. The definitions were as follows:

\section{Normal}

No ultrasound abnormality detected at any time.

\section{Small haemorrhage}

Echoes in and around the region of the germinal matrix and corresponding to the grade I haemorrhage of Levene et al. ${ }^{10}$

\section{Intraventricular haemorrhage}

Echoes within the lateral ventricles with distension or formation of an intraventricular clot. This corresponds to the grade II lesion of Levene et al. ${ }^{10}$

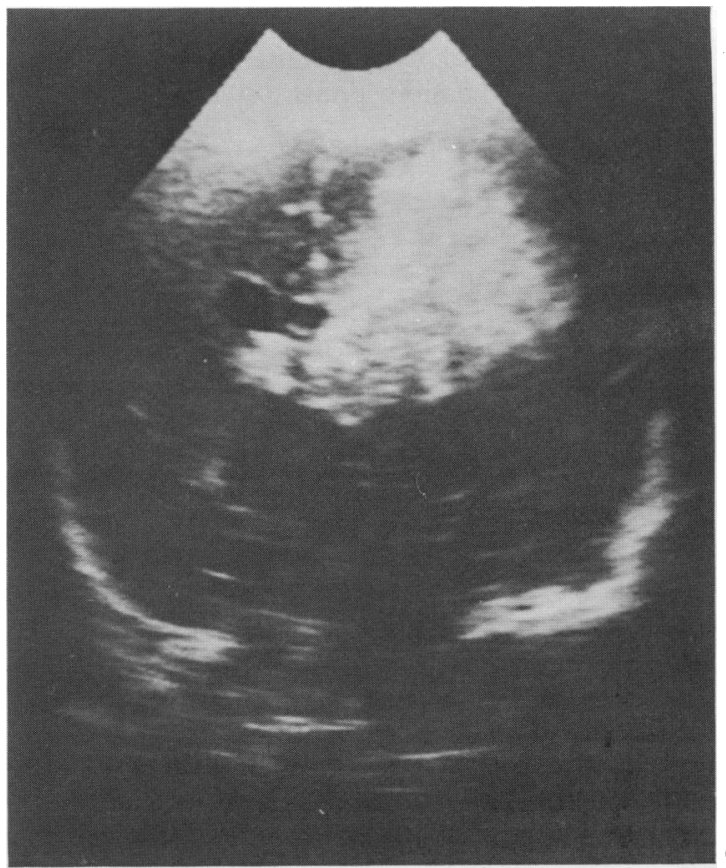

Fig. 1 Parenchymal haemorrhage. Coronal ultrasound scan showing a large echodense lesion involving the right lateral ventricle and parenchyma. There is complete loss of the outline of ventricular structures.

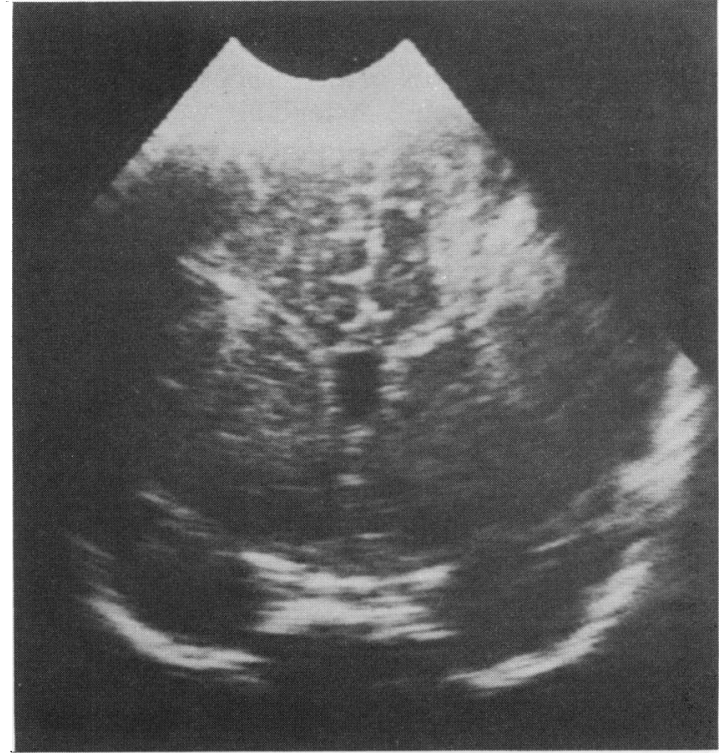

Fig. 2 Periventricular leucomalacia. Coronal ultrasound scan showing a triangular echodense area with its apex lying at the lateral margin of the right lateral ventricle. There is a less extensive, but smaller, echodensity in the left sided parenchyma.

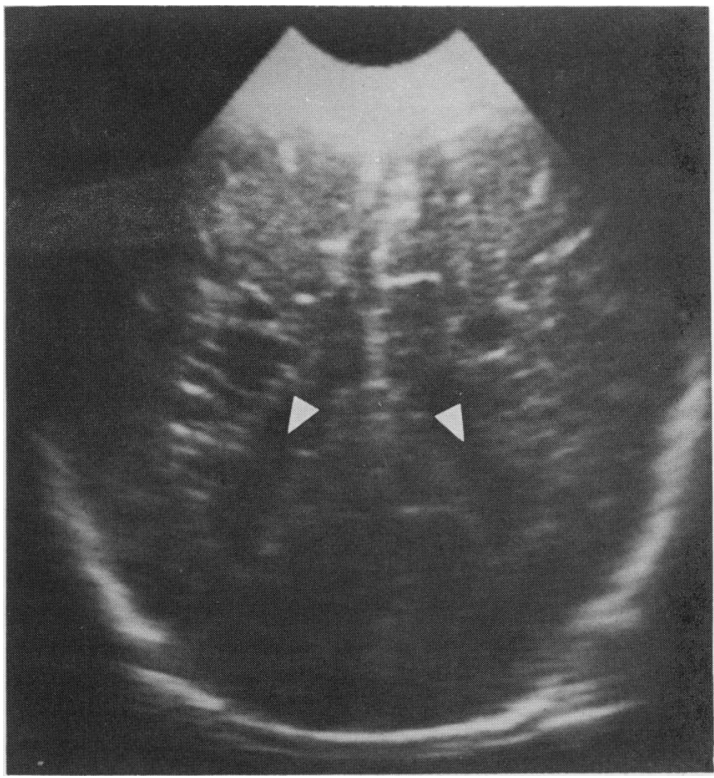

Fig. 3 Cystic degeneration. Posterior coronal ultrasound scan showing bilateral and multiple echofree lesions separate from the lateral ventricles (arrow head). 


\section{Parenchymal haemorrhage}

An echodense area involving the ventricle and the parenchyma. The apex of the echoes lies near the midline with the base extending well into the parencyhmal substance. There is complete loss of the outline of ventricular structures (Fig. 1). In surviving infants a porencephalic cyst would develop with complete loss of the ependyma of the lateral part of the lateral ventricle. We refer to this lesion as representing 'parenchymal haemorrhage'.

\section{Periventricular leucomalacia (PVL)}

We recognised three appearances that we believed represented the range of PVL.

(a) Cystic. An echodense triangle with its apex lying at the lateral border of the lateral ventricle. In many infants intraventricular clot is seen within the ventricle and accompanies this parenchymal appearance. The triangular parenchymal appearance has been recently described by Rushton et al, ${ }^{8}$ and we refer to it as 'PVL' (Fig. 2). In surviving infants the echodensity resolves to discrete echofree cavities representing cyst formation (Fig. 3). The ependyma of the lateral ventricles remains intact in most cases. (b) Precystic. Some infants die in the precystic (echodense) phase, and if the ultrasound appearances described above are present at death this is referred to as precystic PVL.

(c) Prolonged flare. An appearance of relative increased echodensity in the periventricular region seen in both coronal and parasagittal views and persisting for at least two weeks but not undergoing cystic degeneration (Fig. 4). Although subjective assessment of echodensity is difficult, these lesions are less echogenic than those of parenchymal haemorrhage, despite standardised gain and energy output settings.

\section{Ventricular dilatation}

(a) Progressive. Ventricular index rapidly increasing to cross the 97 th centile line. ${ }^{16}$ This usually required treatment with lumbar punctures or a ventricular shunt.

(b) Persistent but non-progressive. Ventricular index increasing more slowly to cross above the 97th centile line but then arresting and following a normal rate of growth.

Babies with ventricular index greater than the $9 \overline{7}$ th centile due to distension with echodense haemorrhage, and who subsequently return to normal measurements of ventricular index, were not included in the ventricular dilatation group.

\section{Results}

Of the 209 babies of birth weight $\leqslant 1500 \mathrm{~g}$, seven were excluded because they died before a scan could be performed and two because they were transferred from outlying hospitals too late for a scan to
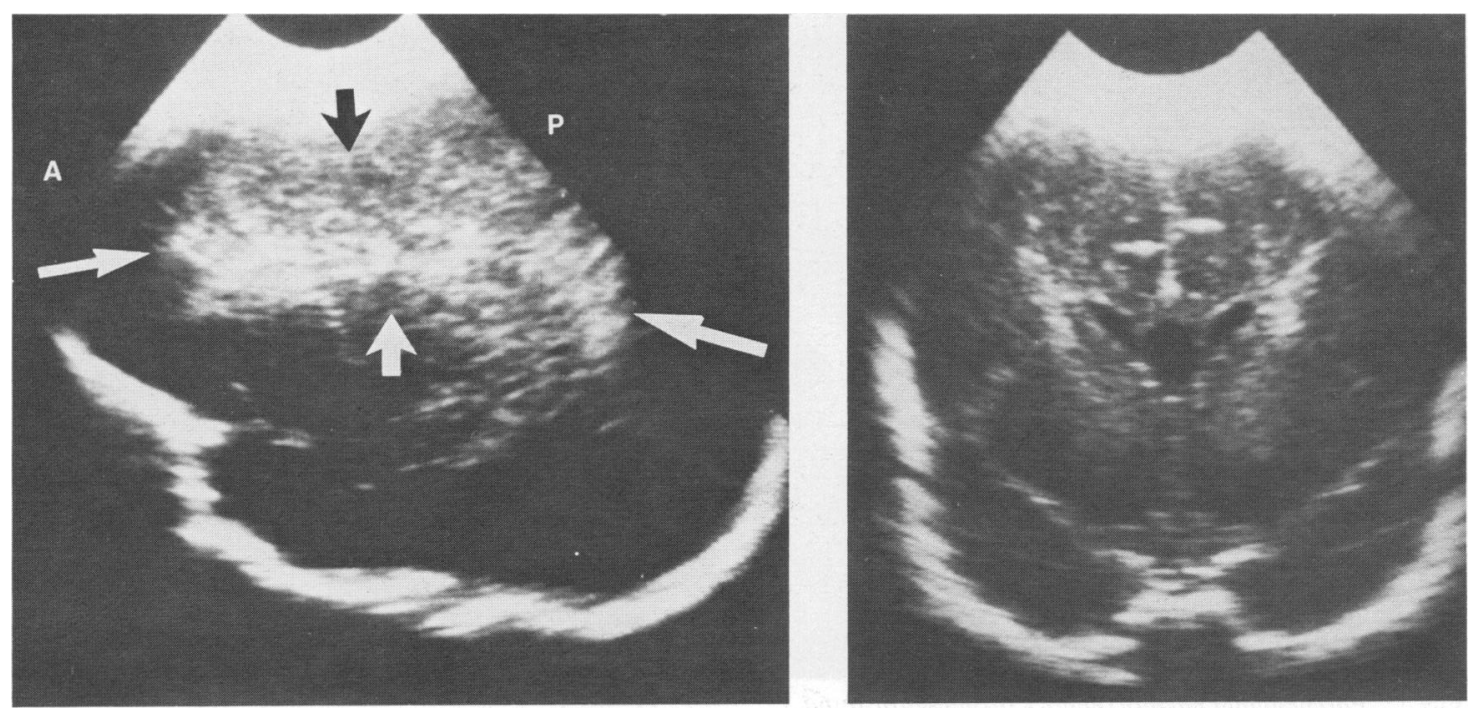

Fig. 4 Echodense flare. Coronal ultrasound scan (right) showing discrete bilateral echodense areas in the periventricular region. There is no intraventricular haemorrhage. Parasagittal scan (left) showing extent of the flare (arrows) extending from the frontal region to the occipital region. $A=A$ Anterior, $P=$ posterior. 
be performed within the first four days of life. The remaining two hundred infants form the subject of this report. Median birth weight was $1200 \mathrm{~g}$ (range 650-1500 g) and median gestational age was 29 weeks (range 25-37 weeks). Fifty six infants had a birth weight $\leqslant 1000 \mathrm{~g}$.

Sixty nine infants $(34 \%)$ had entirely normal scans.

Haemorrhage. Haemorrhage was seen in 107 babies $(53 \%)$, and in 37 this was in the region of the germinal matrix (grade I haemorrhage). Grade II lesions occurred in 62 babies, and in a further eight there was parenchymal haemorrhage.

Parenchymal haemorrhage and PVL. Of the eight babies with parenchymal haemorrhage on early scans, four subsequently developed echodensity in the contralateral periventricular region after a variable period (Fig. 5). In the two long term survivors the former lesion resolved, with the formation of a large porencephalic cyst, and the latter into multiple discrete cysts. We believe this appearance represents parenchymal haemorrhage on one side with

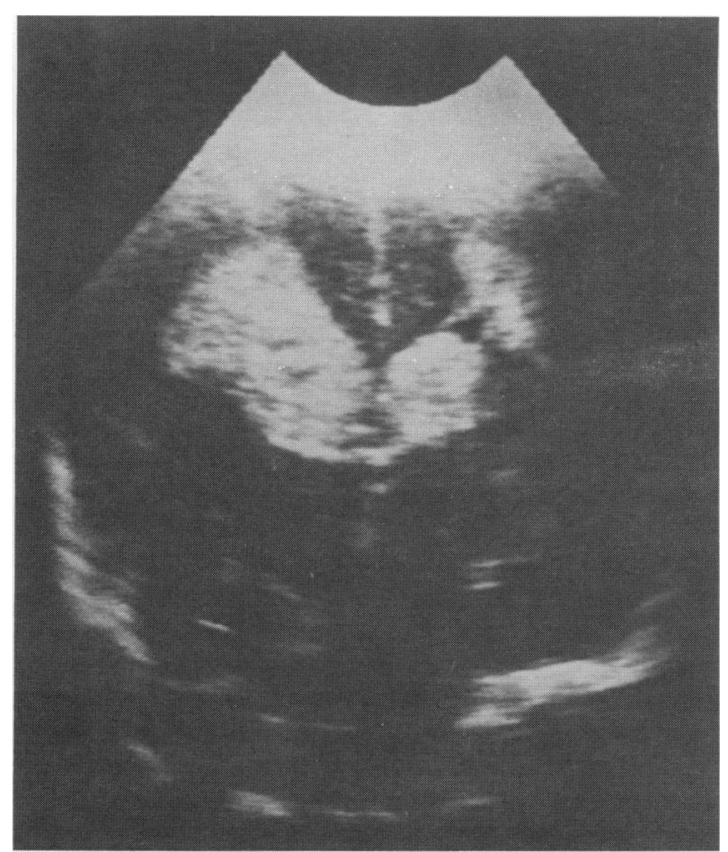

Fig. 5 Parenchymal haemorrhage with contralateral periventricular leucomalacia. Coronal ultrasound scan showing extensive haemorrhage in both lateral ventricles with parenchymal extension on the left side. There is an echodense triangular lesion in the right periventricular area, which developed some time after the initial haemorrhage. contralateral PVL. One infant had a prolonged flare of the opposite side; in only three babies with parenchymal haemorrhage was there no associated contralateral parenchymal lesion.

PVL. Fifty two babies (26\%) showed parenchymal lesions thought not to be primarily haemorrhagic. Eight babies died in the precystic phase of PVL, and in a further 19 infants cysts subsequently developed. In another 25 there was prolonged flare of periventricular echodensity.

A total of 123 infants had evidence of haemorrhage, cystic or precystic PVL, or flare (Fig. 6). Haemorrhage alone occurred in 71 infants, and a further 36 had the appearances' of PVL together with haemorrhage. Fifty five infants had parenchymal involvement (Fig. 7), and only three of these showed the appearances of haemorrhage alone. Two others had PVL alone and 14 had a flare with no other abnormality. Thirty six of the $55(65 \%)$ showed evidence of both haemorrhage and PVL.

Ventricular dilatation. Twenty one babies $(10 \%)$ developed ventricular dilatation; nine were nonprogressive and 12 required treatment. Twelve of the $21(57 \%)$ had associated parenchymal involvement. Thirteen infants developed ventricular dilatation by 14 days, and in all cases it had developed by the age of 4 weeks.

Deaths. Forty two babies (21\%) died, and all deaths occurred before discharge from hospital. Twenty one of the babies who died weighed $\leqslant 1000 \mathrm{~g}$ at birth. Fourteen of the deaths involved babies of 26 weeks' gestation or less. The time of death ranged from day 1 to 121 , and 22 of the babies died before 1 week of age. Postmortem information was obtained in 30 of the $42(71 \%)$ dead infants and is discussed in a paper on pathological correlation. ${ }^{17}$

Timing. Haemorrhage occurred within the first seven days of life in 83 of the $107(78 \%)$ infants, but 17 infants $(15 \%)$ showed the first ultrasound appearance of haemorrhage after 2 weeks of age. Nine of the $107(8 \%)$ babies with haemorrhage did not show an abnormality until after 31 days of age.

The echodense phase of PVL was first noted by the age of 3 days in 14 babies and by 1 week in a further 11. In the final two infants the change was observed by the 10th day.

Nineteen infants developed cysts. One was present at the first scan at age 2 days. Cysts developed between 4 and 7 days in four and after 11 days in the rest. The median time for cysts to be first detected was 18 days of age. Only one infant developed an echofree cystic lesion after 30 days of age. 


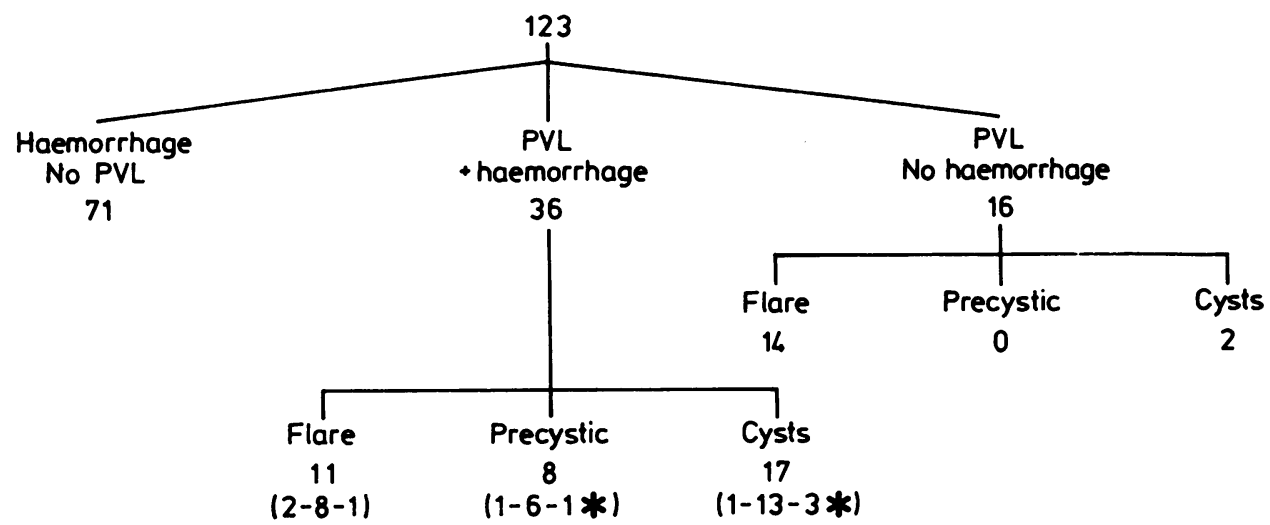

Fig. 6 Distribution of haemorrhage and periventricular leucomalacia. Figures in brackets refers to the numbers of infants with germinal matrix, intraventricular, and parenchymal haemorrhage, respectively.

*Four babies extension one side. periventricular leucomalacia the other.

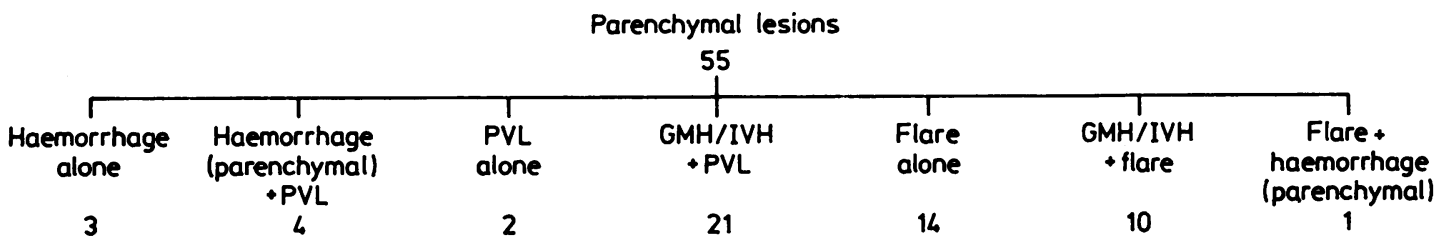

Fig. 7 The patterns of parenchymal lesions. GMH=Germinal matrix haemorrhage; IVH=intraventricular haemorrhage; $P V L=$ periventricular leucomalacia.

Eleven of the $25(44 \%)$ infants with prolonged flares were found to have this abnormality on the first scan (1-3 days). Eighteen ( $72 \%$ ) developed flaring by the end of the first week. The flare was first noted after 20 days in only two infants.

Gestation. Of the 107 babies with haemorrhage, 99 $(93 \%)$ were of 30 weeks' gestation or less. There was a pronounced decrease in the prevalence of haemorrhage from $62 \%$ at 30 weeks' to $19 \%$ at 31 weeks' gestation. A similar pattern was seen in the babies with PVL and prolonged flare: 25 out of 27 babies with PVL and 22 out of 25 babies with prolonged flare had a gestation of 30 weeks or less.

\section{Discussion}

The incidence of the ultrasound appearances that we believe represent cystic and precystic PVL is $13.5 \%$ in a group of very low birthweight infants. A previous retrospective study from the Hammersmith Hospital reported an incidence of $7 \cdot 5 \%{ }^{4}$ and Fawer et al found the incidence to be $16.8 \%$ among relatively few infants of 34 weeks' gestation or below. ${ }^{5}$ Bozynski and colleagues found cystic PVL in $5 \%$ of babies of birth weight $\leqslant 1200 \mathrm{~g} .^{14}$ They studied only surviving infants and this is therefore not an accurate reflection of the overall incidence of the condition. Sinha and colleagues have recently reported the incidence of haemorrhage and ischaemia in a group of premature infants of 32 weeks' gestation or below. ${ }^{11}$ Almost $18 \%$ of their infants showed ultrasound appearances of PVL. The only other published incidence of PVL derives from postmortem studies and varies between $7 \%$ and $34 \% .{ }^{19}$ The latter study looked only at a highly selected group of 106 infants of birth weight $\leqslant 1500 \mathrm{~g}$, all of whom were outborn. Computed tomography has proved to be disappointing in its ability to diagnose hypoxic-ischaemic lesions in the premature neonate ${ }^{2021}$ and compares poorly with real time ultrasound.

All areas of extensive echodensity within the parenchyma seen in association with intraventricular haemorrhage have until recently been assumed to be parenchymal haemorrhage. Hill et al were the first to recognise that parenchymal echodensity might be due to haemorrhagic infarction, ${ }^{3}$ and Levene and 
colleagues later reported the evolution of 'haemorrhagic' PVL to cystic degeneration. ${ }^{4}$ Nwaesei and coworkers have shown that echodensity may be due to gliosis without haemorrhage. ${ }^{9}$ Few would now disagree that multiple small echolucent areas in the periventricular white matter represent PVL. A characteristic triangular echodense region in the parenchyma precedes cystic degeneration, and if seen in those infants who died before cysts develop then we have termed this the 'precystic' phase of PVL. Rushton reports accurate pathological correlation of PVL in five infants with the precystic ultrasound appearance ${ }^{8}$ and we have confirmed this. ${ }^{17}$

The most difficult and controversial area of the diagnosis of parenchymal lesions is the differentiation between extension of haemorrhage and PVL. Even at autopsy it may be impossible to distinguish primary haemorrhage from bleeding into an ischaemic lesion or venous infarction. Armstrong and Norman showed bleeding into an ischaemic periventricular area in $25 \%$ of their cases. ${ }^{18}$ Flodmark and colleagues reported that of 19 preterm babies thought to have parenchymal haemorrhage on computed tomography, autopsy revealed bleeding into an ischaemic area in $18(95 \%) .{ }^{21}$ More recently Volpe and his colleagues, using positron emission tomography, showed extensive areas of diminished cerebral blood flow around an apparent primary parenchymal haemorrhage and suggested that the haemorrhage may have been secondary to ischaemia. ${ }^{22}$ These reports do not prove that ischaemia is the underlying cause of parenchymal haemorrhage in all cases (as suggested by Rushton and colleagues), but they do make it likely that a number of such lesions are primarily ischaemic.

The appearance we refer to as 'parenchymal haemrrrhage' and the subsequent evolution to porencephaly is quite different from that which we recognise as PVL (see Figs. 1 and 2). We have seen four infants in whom the appearance of parenchymal haemorrhage preceded contralateral PVL, and the two survivors developed porencephaly in one hemisphere and multiple cystic degeneration in the other. The aetiology of the underlying cause of these two lesions is, we believe, quite different. The timing also suggests that at least in some cases the massive parenchymal haemorrhage may induce secondary cerebral ischaemia with contralateral PVL. The delayed timing of the parenchymal echodensity after intraventricular haemorrhage also suggests venous infarction as the possible cause. Only eight infants showed the appearance we believe to represent parenchymal haemorrhage.

The term PVL was originally coined by Banker and Larroche to describe a specific but subtle macroscopic appearance in infants dying after a complicated perinatal course. ${ }^{23}$ The most obvious lesions were 'white spots'. Since then the term PVL has been used more widely to describe a number of different macroscopic and microscopic appearances. ${ }^{24} 25$ Although the aetiology of PVL may be border zone infarction, more than one aetiological factor is probably involved. We believe that some cases may be due to venous infarction: eight babies in our series showed a pattern of thrombus distending a lateral ventricle associated with development of a triangular echogenic area in the ipsilateral parenchyma. Pape and Wigglesworth have described congestion of the branches of the terminal vein in cases of massive germinal matrix haemorrhage with associated venous infarction of the surrounding periventricular white matter. ${ }^{24}$ This would account for the incidence of PVL in infants with grade II haemorrhage.

Our overall incidence of haemorrhage $(53 \%)$ corresponds with previous studies of the very low birthweight infant. ${ }^{15}$ Of the 107 babies with haemorrhage described here, parenchymal extension occurred in only eight $(7 \%)$, whereas previous workers have reported parenchymal involvement in about $20 \%$ of cases. ${ }^{15} \mathrm{We}$ believe that many parenchymal lesions that we now recognise as PVL have been classified as parenchymal haemorrhages in the past, and this would explain our lower incidence for the parenchymal lesion.

This is the first prospective study of the incidence of abnormal ultrasound appearances that have been carefully defined before starting collection of data. The definition of cystic and precystic PVL was based on sound pathological correlation, but we chose to use the term 'flare' to avoid assuming pathological abnormality in all cases where this was prolonged. Some periventricular echodensity is very common ${ }^{26}$ and usually transient, and we elected to include as abnormal only those patients in whom the echoes persisted for two weeks or more and could be consistently seen in two planes. Our subsequent autopsy correlation with prolonged flares in three hemispheres confirmed extensive gliosis and microcalcification $^{17}$ and agrees with the data of Nwaesei $e t$ al. ${ }^{9}$ Prolonged flare was seen in $12 \%$ of infants in our study and is clearly an important ultrasound finding, but we must await follow up data on the outcome of these infants before establishing its prognostic importance.

Previous studies have shown that most intraventricular haemorrhages occur within three days of birth. ${ }^{15} 27$ In this study accurate timing of intraventricular haemorrhage was not possible, but $78 \%$ of the haemorrhages were noted before 7 days 
of age. Nine of the 107 haemorrhages $(8 \%)$ occurred after 1 month of age. In one case the haemorrhage was first noted the day after a cardiac arrest, but in the other eight there was no preceding insult. This group resembles the pattern of late haemorrhages as reported by Hecht et al, ${ }^{28}$ and like his cases the haemorrhages in our babies were small. The echodense phase of PVL was observed by 3 days of age in 14 cases and by 10 days of age in the other 13 . This contrasts with the study of the Toronto group in which the mean age for first noting the echodensity was 37 days (range 1-85). ${ }^{9}$ The difference may be partly due to their selection criteria as the babies had to survive at least 20 days for inclusion in their retrospective study.

In conclusion, we report an incidence of periventricular leucomalacia, precystic and cystic, of $13.5 \%$ in a cohort of 200 babies of birth weight $\leqslant 1500 \mathrm{~g}$. The appearance of a prolonged flare, which we argue also represents PVL, occurred in a further 25 infants $(12 \cdot 5 \%)$. Overall, the ultrasound appearances of PVL when prolonged flares are included in this group occurred in 52 of the 200 infants, representing an incidence of $26 \%$ of very low birthweight infants. This is considerably higher than any previous report.

The authors are grateful for the help and cooperation of the medical and nursing staff in both centres and to the Department of Medical Illustration, Leicester Royal Infirmary. Dr Trounce is supported by the Spastics Socicty.

\section{References}

' Clark CE, Clyman RI, Roth RS, Sniderman SH, Lane B, Ballard RA. Risk factor analysis of intraventricular hemorrhage in low-birth-weight infants. J Pediatr 1981;99:625-8.

2 Bejar R, Curbelo V, Coen RW, Leopold G, James H, Gluck L. Diagnosis and follow-up of intraventricular and intracerebral hemorrhages by ultrasound studies of infant's brain through the fontanelles and sutures. Pediatrics 1980;66:661-73.

${ }^{3}$ Hill A, Melson GL, Clark HB, Volpe JJ. Hemorrhagic periventricular leukomalacia: diagnosis by real time ultrasound and correlation with autopsy findings. Pediatrics 1982;69:282-4.

${ }^{4}$ Levene MI, Wigglesworth JS, Dubowitz V. Hemorrhagic periventricular leukomalacia in the neonate: a real-time ultrasound study. Pediatrics 1983;71:794-7.

5 Fawer C-L, Calame A, Perentes E, Anderegg A. Periventricular leukomalacia: a correlation study between realtime ultrasound and autopsy findings. Neuroradiology 1985;27:292-300.

6 Bowerman RA, Donn SM, DiPietro MA, D'Amato CJ, Hicks SP. Periventricular leukomalacia in the pre-term newborn infant: sonographic and clinical features. Radiology 1984;151:383-8.

${ }^{7}$ De Vries LS, Dubowitz LMS, Dubowitz V, et al. Predictive value of cranial ultrasound in the newborn baby: a reappraisal. Lancet 1985;ii:137-40.

${ }^{8}$ Rushton DI, Preston PR, Durbin GM. Structure and evolution of echo dense lesions in the neonatal brain. Arch Dis Child 1985;60:798-808.
${ }^{9}$ Nwaesei CG, Pape KE, Martin DJ, Becker LE, Fitz CR. Periventricular infarction diagnosed by ultrasound: a postmortem correlation. J Pediatr 1984;105:106-10.

${ }^{10}$ Levene MI, Williams JL, Fawer C-L. Ultrasound of the infant brain. Oxford: Blackwell Scientific Publications (Spastics International Medical Publications), 1985.

"Sinha SK, Davies JM, Sims DG, Chiswick ML. Relation between periventricular haemorrhage and ischaemic brain lesions diagnosed by ultrasound in very pre-term infants. Lancet 1985;ii:1154-6.

12 Levene MI, Dubowitz LMS, de Crespigny LCh. Classifying intraventricular haemorrhage. Lancet 1983; ii:49.

${ }^{13}$ Dubowitz LMS, Bydder GM, Mushin J. Developmental sequence of periventricular leukomalacia. Arch Dis Child 1985;60:349-55.

14 Bozynski MEA, Nelson MN, Matalon TAS, et al. Cavitary periventricular leukomalacia: incidence and short-term outcome in infants weighing $<1200$ grams at birth. Dev Med Child Neurol 1985;27:572-7.

15 Levene MI, Fawer C-L, Lamont RF. Risk factors in the development of intraventricular haemorrhage in the preterm neonate. Arch Dis Child 1982;57:410-7.

16 Levene MI. Measurement of the growth of the lateral ventricles in preterm infants with real-time ultrasound. Arch Dis Child 1981;56:900-4.

1) Trounce JQ, Fagan D, Levene MI. Intraventricular haemorrhage and periventricular leucomalacia: ultrasound and autopsy correlation. Arch Dis Child 1986;61:1203-7.

18 Armstrong D, Norman MG. Periventricular leucomalacia in neonates. Arch Dis Child 1974;:49:367-75.

${ }^{19}$ Pape KE, Armstrong DL, Fitzhardinge PM. Central nervous system pathology associated with mask ventilation in the vero low birthweight infant: a new etiology for intracerebellof hemorrhages. Pediatrics 1976;58:473-83.

20) Estrada M, El Gammal T, Dyken PR. Periventricular lo attenuations. Arch Neurol 1980;37:754-6.

${ }^{21}$ Flodmark O, Becker LE, Harwood-Nash DC, Fitzhardinge PM Fitz CR, Chuang SH. Correlation between computed tomography and autopsy in premature and full-term neonates that have suffered perinatal asphyxia. Radiology 1980;137:93-103.

22 Volpe JJ, Herscovitch P, Perlman JM, Raichle ME. Positron emission tomography in the newborn. Extensive impairment of regional cerebral blood flow with intraventricular hemorrhage and hemorrhagic intracerebral involvement.' Pediatrics 1983;72:589-601.

${ }^{23}$ Banker BQ, Larroche J-C. Periventricular leukomalacia of infancy. A form of neonatal anoxic encephalopathy. Arch Neurol 1962;7:386-410.

${ }^{24}$ Pape KE, Wigglesworth JS. Haemorrhage, ischaemia and the perinatal brain. London: William Heinemann (Spastics International Medical Publications), 1979.

25 Anonymous. Ischaemia and haemorrhage in the premature brain [Editorial]. Lancet 1984;ii:847-8.

26 Grant EG, Schellinger D, Richardson JD, Coffey ML, Smirniotopoulous JG, Echogenic periventricular halo: normal sonographic finding or neonatal cerebral hemorrhage. $A J R$ 1983;140:793-6.

27 De Crespigny LC, Mackay R, Murton LJ, Roy RND, Robinson PH. Timing of neonatal cerebroventricular haemorrhage with $N$ ultrasound. Arch Dis Child 1982;57:231-3.

${ }^{28}$ Hecht ST, Filly RA, Callen PW, Wilson-Davis SL. Intracranial hemorrhage: late onset in the preterm neonate. Radiology 1983;149:697-9.

Correspondence to Dr M I Levene, Department of Child Health, Clinical Sciences Buildings, Leicester Royal Infirmary, PO Box 65, Leicester LE2 7LX, England.

Received 28 May 1986 